\title{
Prevalence of hyperthyroidism according to type of vegetarian diet
}

\author{
Serena Tonstad ${ }^{1, *}$,Edward Nathan ${ }^{1}$, Keiji Oda ${ }^{2}$ and Gary E Fraser ${ }^{3}$ \\ 'Department of Health Promotion and Education, School of Public Health, Loma Linda University, 24951 N. Circle \\ Drive, Nichol Hall Room 1519, Loma Linda, CA 92354, USA: ${ }^{2}$ Department of Epidemiology and Biostatistics, \\ School of Public Health, Loma Linda University, Loma Linda, CA, USA: ${ }^{3}$ Department of Cardiology, School of \\ Medicine and Department of Epidemiology and Biostatistics, School of Public Health, Loma Linda University, \\ Loma Linda, CA, USA
}

Submitted 15 November 2013: Final revision received 29 August 2014: Accepted 2 September 2014: First published online 29 September 2014

\begin{abstract}
Objective: Vegetarian diets may be associated with low prevalence of autoimmune disease, as observed in rural sub-Saharan Africans. Graves' disease, an autoimmune disorder, is the most common cause of hyperthyroidism. We studied prevalence of hyperthyroidism according to dietary pattern in a population with a high proportion of vegetarians.

Design: Cross-sectional prevalence study. The association between diet and prevalence of hyperthyroidism was examined using multivariate logistic regression analyses controlling for sociodemographic characteristics and salt use. Setting: The Adventist Health Study-2 conducted in the USA and Canada.

Subjects: Church members ( $n$ 65 981) provided demographic, dietary, lifestyle and medical history data by questionnaire.

Results: The prevalence of self-reported hyperthyroidism was $0.9 \%$. Male gender $(\mathrm{OR}=0.32 ; 95 \% \mathrm{CI} 0 \cdot 26,0.41)$ and moderate or high income (OR $=0.67 ; 95 \% \mathrm{CI}$ $0.52,0.88$ and $\mathrm{OR}=0.73 ; 95 \%$ CI $0.58,0.91$, respectively) protected against hyperthyroidism, while obesity and prevalent CVD were associated with increased risk $(\mathrm{OR}=1.25 ; 95 \% \mathrm{CI} 1.02,1.54$ and $\mathrm{OR}=1.92 ; 95 \% \mathrm{CI} 1 \cdot 53,2 \cdot 42$, respectively). Vegan, lacto-ovo and pesco vegetarian diets were associated with lower risk compared with omnivorous diets ( $\mathrm{OR}=0.49 ; 95 \% \mathrm{CI} 0.33, \mathrm{OR}=0 \cdot 72,0 \cdot 65 ; 95 \% \mathrm{CI}$ $0.53,0.81$ and $\mathrm{OR}=0.74 ; 95 \% \mathrm{CI} 0.56,1.00$, respectively).

Conclusions: Exclusion of all animal foods was associated with half the prevalence of hyperthyroidism compared with omnivorous diets. Lacto-ovo and pesco vegetarian diets were associated with intermediate protection. Further study of potential mechanisms is warranted.
\end{abstract}

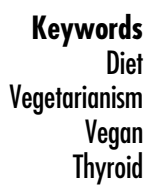

There is growing awareness that a plant-based diet decreases morbidity and mortality associated with a range of chronic disease including hypertension, diabetes and coronary artery disease $^{(1)}$. While diet plays an obvious role in management of autoimmune disease like coeliac disease or type 1 diabetes, fewer studies have documented relationships between diet and autoimmune diseases ${ }^{(2)}$. Isolated rural populations following traditional diets and lifestyles appear to be protected from autoimmune disease. Observations by Trowell five decades ago indicated that in rural sub-Saharan Africans consuming a near-vegan diet a number of autoimmune disorders were rare, or virtually unknown, including rheumatoid arthritis, type 1 diabetes, thyrotoxicosis and Hashimoto's thyroiditis ${ }^{(2)}$.

The effects of plant-based diets may be due to the exclusion of animal foods, the effects of plant foods, or both.
Animal foods like meat, eggs and dairy products may contain high oestrogen concentrations, which have been linked to autoimmunity in cell and animal studies ${ }^{(3)}$. Small studies have found that fasting followed by a vegetarian or a Mediterranean diet rich in vegetables and oils may ameliorate symptoms of rheumatoid arthritis; however, effects are still uncertain due to the small number of studies ${ }^{(4)}$.

Hyperthyroidism is a prevalent condition with many causes, of which the most common is Graves' disease ${ }^{(5)}$. Graves' disease is an autoimmune disorder caused by antibodies directed against the thyrotropin receptor, resulting in excess synthesis and secretion of thyroid hormone. Dietary causes of hyperthyroidism are rare, although excess dietary iodine has been linked to hyperthyroidism $^{(6,7)}$. Rarely, contaminated meat products have been reported as a cause of thyrotoxicosis ${ }^{(8,9)}$. 
In the current study we hypothesized that vegetarian diets may protect against hyperthyroidism in a Western population. We compared the prevalence of hyperthyroidism according to dietary pattern in a population exhibiting a wide range of diets. These diets included a vegan diet that excluded all animal products, a lacto-ovo vegetarian diet that excluded meat but allowed milk and eggs, a pesco vegetarian diet that included fish but no meat and a nonvegetarian diet.

\section{Methods}

\section{Participants and study design}

The Adventist Health Study-2 (AHS-2) is a longitudinal study initiated to investigate the role of foods and their relationship to disease, particularly cancer. Participants were members of the Seventh-day Adventist church recruited through their respective churches in the USA and Canada. About 97000 individuals joined between 2002 and $2006^{(10)}$. Participants were eligible if they were proficient in English and were aged 30 years or older. All instruments and procedures were approved by the Loma Linda University Institutional Review Board in June 2001; approval was renewed annually thereafter.

\section{Questionnaire}

Once enrolled, each participant received a previously validated questionnaire with the informed consent materials. The questionnaire was divided into sections on demographics including place of early residence, medical history, dietary patterns, physical activity and other lifestyle habits and self-reported height and weight. Questionnaires were returned by pre-paid envelopes and edited for missing data and stray marks. If any of the research staff felt data entries required confirmation, or demographic data were missing, a telephone call was made to verify these entries.

Race and ethnicity were elicited for the following categories: Black/African American, West Indian/Caribbean, African and other Black, Hispanic and non-Hispanic White. In the current study all Blacks were collapsed into one category with non-Blacks in the other category. Respondents reported annual personal and household income by checking one of eight income categories, ranging from <\$US 10000 to $>$ \$US 200000 . Responses were grouped into four categories: $\leq$ WUS 10000 , \$US 11000 20000 , \$US $21000-30000$ and >\$US 30000 . BMI was calculated from respondents' self-reported height and weight as $\mathrm{kg} / \mathrm{m}^{2}$. These self-reports were validated ${ }^{(11)}$.

\section{Prevalent cases of hyperthyroidism and other diseases}

In the medical history section of the questionnaire, respondents were asked if they had hyperthyroidism diagnosed by a physician and if affirmative to indicate when they were first diagnosed. They were also asked if they have been treated for hyperthyroidism within the last 12 months. Prevalent CVD included all cases of reported angina, heart attack, stroke and transient ischaemic attack.

\section{Dietary information}

The FFQ used in AHS-2 includes over 200 hard-coded foods and space for fifty write-ins covering the diet during the past 12 months ${ }^{(12)}$. Vegetarian status was categorized by defining: vegans as participants who reported consuming no animal products (red meat, poultry, fish, eggs, milk and dairy products $<1$ time/month); lacto-ovo vegetarians as those who consumed dairy products and/or eggs but no fish or meat (red meat, poultry and fish $<1$ time/month); pesco vegetarians as those who consumed fish $\geq 1$ time/month and dairy products and/or eggs but no red meat or poultry (red meat and poultry $<1$ time/ month); semi-vegetarians as those who consumed dairy products and/or eggs and meat (red meat and poultry $\geq 1$ time/month and $<1$ time/week); and omnivores as those who consumed animal products (red meat, poultry, fish, eggs, milk and dairy products $>1$ time/week). The frequency of adding salt to food was queried as $\leq 1$ time/ week, 2-6 times/week and $\geq 1$ time/d. Iodine intake was not specified in the questionnaire. A previous report described the dietary methods and nutrient profiles of the vegetarian and non-vegetarian dietary patterns in the study in more detail ${ }^{(12)}$. In summary, lacto-ovo vegetarians had lower intakes of dairy fat and animal protein than nonvegetarians while vegans had the lowest intakes of saturated and trans-fat and arachidonic acid, and the highest intakes of fibre, soya protein, $\beta$-carotene and Mg. Lactoovo and pesco vegetarians had intermediate intakes of saturated fat, fibre, soya protein, $\beta$-carotene and $\mathrm{Mg}$.

To validate the nutrient intakes, a calibration study was conducted among participants randomly selected from the AHS-2 cohort $^{(13,14)}$. The result showed that the validity coefficients were moderate to high for macronutrients, fatty acids, vitamins, minerals and fibre.

\section{Statistical analyses}

Participants who reported treatment for hyperthyroidism in the past 12 months were a priori considered prevalent cases in the primary statistical analyses ( $n$ 603). We conducted a sensitivity analysis that included participants who reported treated hyperthyroidism, but not in the past 12 months.

Descriptive characteristics of the population were compared between participants with and without hyperthyroidism and between dietary patterns (vegan, lacto-ovo vegetarian, pesco vegetarian, semi-vegetarian or nonvegetarian) using the $\chi^{2}$ test, the two-sample independent $t$ test or ANOVA, as appropriate. Logistic regression was used to examine factors associated with the outcome (prevalence of hyperthyroidism). The logistic regression model included gender, ethnicity, age (as a continuous variable), BMI (categorized as <25.0, 25.0-29.9 and 
Table 1 Demographic characteristics and diet according to prevalence of hyperthyroidism ( $n$ 65981), Adventist Health Study-2

\begin{tabular}{|c|c|c|c|c|c|}
\hline & \multicolumn{4}{|c|}{ Prevalent hyperthyroidism } & \multirow[b]{3}{*}{$P$ value } \\
\hline & \multicolumn{2}{|c|}{ No ( $n$ 65 378; $99.1 \%)$} & \multicolumn{2}{|c|}{ Yes ( $n$ 603; $0.9 \%)$} & \\
\hline & Mean or \% & SD & Mean or \% & SD & \\
\hline Age (years) & $56 \cdot 7$ & 13.7 & $61 \cdot 0$ & 13.7 & $<0.0001$ \\
\hline $\mathrm{BMl}\left(\mathrm{kg} / \mathrm{m}^{2}\right)$ & $27 \cdot 2$ & 5.9 & $28 \cdot 3$ & 6.4 & $<0.0001$ \\
\hline Gender ( $\%$ female) & 63.1 & - & 84.7 & - & $<0.0001$ \\
\hline Race (\% non-Black) & $75 \cdot 8$ & - & 73.1 & - & 0.13 \\
\hline Income $(\%)$ & & & & & $<0.0001$ \\
\hline$\leq \$$ US 10000 & $19 \cdot 8$ & - & $30 \cdot 2$ & - & \\
\hline \$US $11000-20000$ & $19 \cdot 8$ & - & $26 \cdot 2$ & - & \\
\hline \$US $21000-30000$ & $16 \cdot 8$ & - & 13.9 & - & \\
\hline >\$US 30000 & 43.7 & - & 29.7 & - & \\
\hline Education (\%) & & & & & $<0.0001$ \\
\hline$\leq$ High school & $18 \cdot 6$ & - & $27 \cdot 0$ & - & \\
\hline Some college & $39 \cdot 6$ & - & $42 \cdot 1$ & - & \\
\hline$\geq$ College & 41.8 & - & $30 \cdot 9$ & - & \\
\hline Continent of residence during early life (\%) & & & & & 0.73 \\
\hline USA or Canada & $86 \cdot 4$ & - & $86 \cdot 4$ & - & \\
\hline Asia or Australia & 2.5 & - & 2.7 & - & \\
\hline Other & $11 \cdot 1$ & - & $10 \cdot 9$ & - & \\
\hline CVD (\%) & 7.5 & - & $16 \cdot 9$ & - & $<0.0001$ \\
\hline Salt use (\%) & & & & & 0.16 \\
\hline$\leq 1$ time/week & $34 \cdot 8$ & - & $38 \cdot 3$ & - & \\
\hline 2-6 times/week & 43.2 & - & 39.8 & - & \\
\hline$\geq 1 \mathrm{time} / \mathrm{d}$ & $22 \cdot 0$ & - & 21.9 & - & \\
\hline Dietary pattern (\%) & & & & & $<0.0001$ \\
\hline Vegan & $8 \cdot 2$ & - & $4 \cdot 8$ & - & \\
\hline Lacto-ovo vegetarian & 27.9 & - & $21 \cdot 2$ & - & \\
\hline Semi-vegetarian & $5 \cdot 6$ & - & $5 \cdot 1$ & - & \\
\hline Pesco vegetarian & 9.7 & - & $9 \cdot 0$ & - & \\
\hline Omnivore & $48 \cdot 6$ & - & 59.9 & - & \\
\hline
\end{tabular}

${ }^{*}$ Compared by the independent $t$ test (continuous variables) or the $x^{2}$ test (categorical variables).

$\geq 30.0 \mathrm{~kg} / \mathrm{m}^{2}$ ), income, education, report of CVD, frequency of salt use and dietary pattern. Results were presented as odds ratios (for the logistic model) and 95\% confidence intervals. All analyses were done using the statistical software package SAS version 9.3.

\section{Results}

There were 65981 participants with complete data for all variables. Those who were included were younger (mean age 56.7 years) than those who were excluded (mean age 62.6 years), were more likely to be male $(36.7 \% v .31 .4 \%)$ and had a higher proportion with some college education or longer education $(81 \cdot 3 \% v \cdot 69 \cdot 1 \%)$. Among the 65981 participants, $603(0.9 \%)$ reported prevalent hyperthyroidism treated within the past 12 months. As shown in Table 1, participants with hyperthyroidism were older, had a higher BMI and reported less income and education compared with participants without hyperthyroidism. More women than men reported hyperthyroidism. Prevalence of hyperthyroidism did not differ according to early residence and this variable was not studied further. Those who reported hyperthyroidism were less likely to follow vegan, lacto-ovo, pesco and semi-vegetarian diets compared with those without hyperthyroidism, and had a higher prevalence of an omnivorous diet. As shown in Table 2, sociodemographic characteristics, BMI and prevalence of hyperthyroidism differed significantly according to dietary group. Vegetarians were older, had lower BMI, were less likely to be female, had a higher education and less CVD and hyperthyroidism compared with non-vegetarians.

Table 3 shows multivariate odds ratios for factors associated with a diagnosis of hyperthyroidism. Age, female gender and overweight or obesity were associated with increased risk, while increasing education and income were protective against a diagnosis of hyperthyroidism. Vegan, lacto-ovo vegetarian and pesco vegetarian diets were associated with a lower prevalence of hyperthyroidism than omnivorous diets, while semi-vegetarian diets were not associated with less hyperthyroidism.

Results of the sensitivity analysis showed little change in the odds ratio estimates and significance levels (Table 4). While lacto-ovo vegetarians had lower odds of hyperthyroidism than omnivores, the relationship was no longer seen for vegans and pesco vegetarians.

\section{Discussion}

Our findings indicate that participants consuming a vegan diet had $52 \%$ lower odds of prevalent hyperthyroidism 
Table 2 Demographic characteristics and prevalent hyperthyroidism according to dietary pattern ( $n$ 65981), Adventist Health Study-2

\begin{tabular}{|c|c|c|c|c|c|c|c|c|c|c|c|}
\hline & \multicolumn{2}{|c|}{$\begin{array}{c}\text { Vegan } \\
(n 5389 ; 8.2 \%)\end{array}$} & \multicolumn{2}{|c|}{$\begin{array}{c}\text { Lacto-ovo } \\
\text { vegetarian } \\
\text { (n 18390; } 27.9 \%) \\
\end{array}$} & \multicolumn{2}{|c|}{$\begin{array}{l}\text { Semi-vegetarian } \\
(n 3681 ; 5.6 \%) \\
\end{array}$} & \multicolumn{2}{|c|}{$\begin{array}{c}\text { Pesco } \\
\text { vegetarian } \\
(n 6420 ; 9.7 \%) \\
\end{array}$} & \multicolumn{2}{|c|}{$\begin{array}{c}\text { Omnivore } \\
\text { (n } 32101 ; 48.7 \%)\end{array}$} & \multirow[b]{2}{*}{$P$ value } \\
\hline & Mean or $\%$ & SD & Mean or $\%$ & SD & Mean or $\%$ & SD & Mean or $\%$ & SD & Mean or $\%$ & SD & \\
\hline Age (years) & 58.5 & $13 \cdot 8$ & $58 \cdot 0$ & $14 \cdot 0$ & 57.9 & $14 \cdot 1$ & $57 \cdot 4$ & 13.8 & 55.4 & $13 \cdot 2$ & $<0.0001$ \\
\hline $\mathrm{BMI}\left(\mathrm{kg} / \mathrm{m}^{2}\right)$ & 24.0 & 4.6 & $25 \cdot 9$ & $5 \cdot 1$ & $27 \cdot 3$ & 5.6 & $26 \cdot 3$ & $5 \cdot 1$ & $28 \cdot 8$ & $6 \cdot 3$ & $<0.0001$ \\
\hline Gender (\% female) & 60.5 & - & $62 \cdot 2$ & - & $65 \cdot 8$ & - & $66 \cdot 0$ & - & 63.5 & - & $<0.0001$ \\
\hline Race (\% non-Black) & $81 \cdot 2$ & _ & 88.3 & _- & 84.6 & _- & 64.0 & - & $69 \cdot 0$ & _- & $<0.0001$ \\
\hline Income (\%) & & & & & & & & & & & $<0.0001$ \\
\hline$\leq \$$ US 10000 & $27 \cdot 1$ & - & $20 \cdot 1$ & - & $21 \cdot 0$ & - & $17 \cdot 9$ & - & $18 \cdot 9$ & - & \\
\hline \$US $11000-20000$ & $22 \cdot 7$ & - & $19 \cdot 1$ & - & $20 \cdot 6$ & - & $18 \cdot 3$ & - & $20 \cdot 0$ & - & \\
\hline \$US $21000-30000$ & $15 \cdot 8$ & - & $15 \cdot 9$ & - & $16 \cdot 6$ & - & $16 \cdot 2$ & - & 17.5 & - & \\
\hline$>\$$ US 30000 & 34.4 & - & 44.8 & - & 41.8 & - & 47.7 & - & 43.7 & - & \\
\hline Education (\%) & & & & & & & & & & & $<0.0001$ \\
\hline$\leq$ High school & $16 \cdot 7$ & - & $13 \cdot 3$ & - & $20 \cdot 6$ & - & $17 \cdot 7$ & - & $22 \cdot 1$ & - & \\
\hline Some college & 38.7 & - & 34.4 & - & $39 \cdot 3$ & - & 38.2 & - & $43 \cdot 1$ & - & \\
\hline$\geq$ College & $44 \cdot 6$ & - & $52 \cdot 3$ & - & $40 \cdot 1$ & - & $44 \cdot 2$ & - & $34 \cdot 8$ & - & \\
\hline Salt use (\%) & & & & & & & & & & & $<0.0001$ \\
\hline$\leq 1$ time/week & $32 \cdot 8$ & - & $31 \cdot 3$ & - & 37.4 & - & $40 \cdot 5$ & - & $35 \cdot 8$ & - & \\
\hline $2-6$ times/week & $42 \cdot 9$ & - & $45 \cdot 1$ & - & 43.3 & - & 40.9 & - & $42 \cdot 5$ & - & \\
\hline$\geq 1$ time $/ \mathrm{d}$ & $24 \cdot 3$ & - & 23.6 & - & $19 \cdot 4$ & - & $18 \cdot 7$ & - & 21.7 & - & \\
\hline CVD $(\%)$ & 6.5 & - & 6.6 & - & 8.5 & - & $7 \cdot 1$ & - & $8 \cdot 3$ & - & $<0.0001$ \\
\hline Prevalent hyperthyroidism (\%) & 0.5 & - & 0.7 & - & 0.8 & - & 0.8 & - & $1 \cdot 1$ & - & $<0.0001$ \\
\hline
\end{tabular}

${ }^{*}$ Compared by ANOVA (continuous variables) or the $x^{2}$ test (categorical variables).

Table 3 Odds ratios and $95 \%$ confidence intervals for prevalence of hyperthyroidism treated within the past 12 months (603 cases, total $n 65981$ ), Adventist Health Study-2. Odds ratios are adjusted for all of the variables shown

\begin{tabular}{|c|c|c|}
\hline & OR & $95 \% \mathrm{Cl}$ \\
\hline Age (years) & 1.02 & $1.01,1.03$ \\
\hline \multicolumn{3}{|l|}{ Gender } \\
\hline Female & Referent & - \\
\hline Male & 0.32 & $0.26,0.41$ \\
\hline \multicolumn{3}{|l|}{ Race } \\
\hline Non-Black & Referent & - \\
\hline Black & 1.11 & $0.91,1.34$ \\
\hline \multicolumn{3}{|l|}{$\mathrm{BMI}\left(\mathrm{kg} / \mathrm{m}^{2}\right)$} \\
\hline$<25.0$ & Referent & \\
\hline $25 \cdot 0-29.9$ & 1.08 & $0.89,1.32$ \\
\hline$\geq 30.0$ & 1.25 & $1.02,1.54$ \\
\hline \multicolumn{3}{|l|}{ Income } \\
\hline$\leq \$$ US 10000 & Referent & - \\
\hline \$US $11000-20000$ & 0.90 & $0.72,1.11$ \\
\hline \$US $21000-30000$ & 0.67 & $0.52,0.88$ \\
\hline >\$US 30000 & 0.73 & $0.58,0.91$ \\
\hline \multicolumn{3}{|l|}{ Education } \\
\hline$\leq$ High school & Referent & - \\
\hline Some college & 0.88 & $0.71,1.07$ \\
\hline$\geq$ College & 0.84 & $0.66,1.06$ \\
\hline \multicolumn{3}{|l|}{ CVD } \\
\hline None & Referent & - \\
\hline Present & 1.92 & $1.53,2.42$ \\
\hline \multicolumn{3}{|l|}{ Salt use } \\
\hline$\leq 1$ time/week & Referent & - \\
\hline 2-6 times/week & 0.88 & $0.73,1.05$ \\
\hline$\geq 1 \mathrm{time} / \mathrm{d}$ & 0.89 & $0.72,1.11$ \\
\hline \multicolumn{3}{|l|}{ Diētary pattern } \\
\hline Vegan & 0.49 & $0.33,0.72$ \\
\hline Lacto-ovo vegetarian & 0.65 & $0.53,0.81$ \\
\hline Semi-vegetarian & 0.71 & $0.49,1.03$ \\
\hline Pesco vegetarian & 0.74 & $0.56,1.00$ \\
\hline Omnivore & Referent & - \\
\hline
\end{tabular}

Table 4 Odds ratios and $95 \%$ confidence intervals for prevalence of hyperthyroidism treated or not treated within the past 12 months (1191 cases, total $n 65981$ ), Adventist Health Study-2. Odds ratios are adjusted for all of the variables shown

\begin{tabular}{|c|c|c|}
\hline & OR & $95 \% \mathrm{Cl}$ \\
\hline Age (years) & 1.02 & $1.01,1.02$ \\
\hline \multicolumn{3}{|l|}{ Gender } \\
\hline Female & Referent & - \\
\hline Male & 0.31 & $0.27,0.37$ \\
\hline \multicolumn{3}{|l|}{ Race } \\
\hline Non-Black & Referent & - \\
\hline Black & 1.08 & $0.94,1.24$ \\
\hline \multicolumn{3}{|l|}{ BMI $\left(\mathrm{kg} / \mathrm{m}^{2}\right)$} \\
\hline$<25.0$ & Referent & - \\
\hline $25 \cdot 0-29 \cdot 9$ & 0.96 & $0.84,1.11$ \\
\hline$\geq 30 \cdot 0$ & 1.07 & $0.93,1.24$ \\
\hline \multicolumn{3}{|l|}{ Income } \\
\hline$\leq \$$ US 10000 & Referent & - \\
\hline \$US $11000-20000$ & 1.00 & $0.85,1.18$ \\
\hline \$US $21000-30000$ & 0.81 & $0.67,0.98$ \\
\hline$>\$$ US 30000 & 0.91 & $0.77,1.07$ \\
\hline \multicolumn{3}{|l|}{ Education } \\
\hline$\leq$ High school & Referent & - \\
\hline Some college & 1.00 & $0.86,1.17$ \\
\hline$\geq$ College & 1.01 & $0.85,1.20$ \\
\hline \multicolumn{3}{|l|}{ CVD } \\
\hline None & Referent & - \\
\hline Present & 1.63 & $1.36,1.95$ \\
\hline \multicolumn{3}{|l|}{ Salt use } \\
\hline$\leq 1$ time/week & Referent & - \\
\hline 2-6 times/week & 0.92 & $0.81,1.05$ \\
\hline$\geq 1$ time/d & 0.97 & $0.83,1.14$ \\
\hline \multicolumn{3}{|l|}{ Dietary pattern } \\
\hline Vegan & 0.89 & $0 \cdot 71,1 \cdot 11$ \\
\hline Lacto-ovo vegetarian & 0.77 & $0.66,0.89$ \\
\hline Semi-vegetarian & 0.83 & $0.64,1.08$ \\
\hline Pesco vegetarian & 1.03 & $0.85,1.24$ \\
\hline Omnivore & Referent & - \\
\hline
\end{tabular}


compared with omnivores. Lacto-ovo vegetarians and pesco vegetarians also carried lower odds of hyperthyroidism, but the reduction appeared smaller than that in the vegan group. Semi-vegetarian diets were not protective against hyperthyroidism. The strengths of the study include the sizeable cohort following a range of diets who belonged to a church that discourages use of animal foods, smoking and use of alcohol. Thus, cigarette smoking, a common confounder of diet and disease relationships, was extremely rare $(<2 \%)$.

Prevalent cases of hyperthyroidism composed about $0.9 \%$ of the population, which is somewhat lower than national estimates. In comparison, the third National Health and Nutrition Examination Survey (NHANES III) showed a prevalence of hyperthyroidism of $1.3 \%{ }^{(15)}$ while in another source the prevalence was stated as $1 \cdot 2 \%^{(16)}$. The demographic predictors were as expected, with age, female gender and overweight and obesity identified as predictors of hyperthyroidism ${ }^{(5)}$.

Putative mechanisms to explain these findings include the lower BMI of vegans and other vegetarians. Although the relationship between diet and hyperthyroidism was found after controlling for BMI, residual confounding may be present. Obesity is associated with autoimmune disease and studies have linked elevated leptin concentrations to autoimmunity $^{(17)}$. Vegetarian diets have been linked to lower body weight and BMI specifically in this cohort ${ }^{(18)}$. Other suggestions include the hypothesis that vegan diets may downregulate systemic insulin-like growth factor-1 activity, a modulator of lymphocyte proliferation and apoptosis $^{(2)}$. Polyphenolic phytochemicals such as flavonoids found in plant foods are thought to protect cells against autoimmune processes ${ }^{(19)}$. Could type of diet or environmental toxins in food affect the microbiome or directly trigger autoimmune disease? Some data support this notion $^{(20,21)}$. It has been speculated that the rise in autoimmune diseases in recent years may be linked to environmental oestrogens, found in a variety of foods, but particularly in meat, eggs and dairy products given exogenous hormones ${ }^{(3)}$. However, further study is needed regarding any causal effects of plant diets or elimination of animal foods on autoimmune disease. The sensitivity analyses that included all participants regardless of treatment in the last year found robust protection associated with the lacto-ovo vegetarian diet, suggesting that the meat content of omnivorous diets may be risky rather than dairy products and eggs. Notably, a recent review found that high consumption of meat was associated with risk of thyroid cancer while dairy products showed no significant association $^{(22)}$. The vegetarian diets contained high amounts of antioxidants, possible protective factors against autoimmune disease ${ }^{(20)}$.

\section{Limitations}

The present data are hypothesis generating, as there are considerable biases in cross-sectional studies. Unmeasured confounding may be present, although we controlled for a number of confounders of the relationship between diet and thyroid disease, including a range of sociodemographic characteristics. The cause of hyperthyroidism was not specified and a number of aetiologies may have been present in the cases reported, although in iodine-replete populations most thyroid disease is autoimmune. We were only able to confirm the diagnosis of hyperthyroidism by direct contact with a small number of respondents (data not shown) due to the elapse of time after inclusion and medical records were not examined. Vegans may be less likely to visit their physician for physical examination as compared with other dietary groups. Individuals may report thyroid problems because of other medical symptoms, like weight loss. However, in our primary analyses only participants who reported treatment in the last 12 months were considered prevalent cases, to reduce over-reporting. The sensitivity analysis, which included all participants who reported hyperthyroidism regardless of treatment or not in the last 12 months, showed similar results for lacto-ovo vegetarians, but not for vegans and pesco vegetarians. A distant history of hyperthyroidism may be less likely to associate with current diet than a current condition. A study suggests high sensitivity of self-report questionnaires for thyroid disorders ${ }^{(23)}$; however, a major limitation of the current study is the selfreported nature of the data. In a cross-sectional study such as the present one, causality cannot be assumed; further study is required, for example a cross-sectional study comparing diets of individuals with and without hyperthyroidism. This church-going population differed from the general population in several aspects, primarily lack of smoking (only 1-2\% were current smokers) and lesser use of alcohol and stimulants, which is discouraged by the church.

While the dietary questionnaires were validated, data on iodine intake were not measured, so we used added salt as a proxy measure. However, the questionnaire did not specify whether it was iodized. We did not record the family history of thyroid disease, which plays a part in the pathogenesis of autoimmune thyroid disease.

\section{Conclusion}

Vegan and other vegetarian diets were associated with protection against prevalent hyperthyroidism.

\section{Acknowledgements}

Financial support: The work was supported in part by the National Institutes of Health/National Cancer Institute (grant number 5U01CA152939). The funder had no role in the design, analysis or writing of this article. Conflict of interest: None. Authorship: G.E.F. is the primary investigator of AHS-2 and participated in study design, analysis and writing of the paper. S.T. initiated the study and participated in analysis, design and writing. E.N. participated 
in design and writing. K.O. performed the statistical analyses. All the authors have seen and approved the final manuscript. Ethics of human subject participation: All instruments and procedures were approved by the Loma Linda University Institutional Review Board in June 2001; approval was renewed annually thereafter.

\section{References}

1. Fraser GE (2009) Vegetarian diets: what do we know of their effects on common chronic diseases? Am J Clin Nutr 89, issue 5, 1607S-1612S.

2. McCarty MF (2001) Upregulation of lymphocyte apoptosis as a strategy for preventing and treating autoimmune disorders: a role for whole-food vegan diets, fish oil and dopamine agonists. Med Hypotheses 57, 258-275.

3. Chighizola C \& Meroni PL (2012) The role of environmental estrogens and autoimmunity. Autoimmun Rev 11, A493-A501.

4. Hagen KB, Byfuglien MG, Falzon L et al. (2009) Dietary interventions for rheumatoid arthritis. Cochrane Dabase Syst Rev issue 1, CD006400.

5. Cooper DS (2003) Hyperthyroidism. Lancet 362, 459-468.

6. Golkowski F, Buziak-Bereza M, Trofimiuk M et al. (2007) Increased prevalence of hyperthyroidism as an early and transient side-effect of implementing iodine prophylaxis. Public Health Nutr 10, 799-802.

7. Stanbury JB, Ermans AE, Bourdoux P et al. (1998) Iodineinduced hyperthyroidism: occurrence and epidemiology. Thyroid 8, 83-100.

8. Hedberg CW, Fishbein DB, Janssen RS et al. (1987) An outbreak of thyrotoxicosis caused by the consumption of bovine thyroid gland in ground beef. $N$ Engl J Med $\mathbf{3 1 6}$ 993-998.

9. Hendriks LE \& Looij BJ (2010) Hyperthyroidism caused by excessive consumption of sausages. Neth J Med $\mathbf{6 8}$, 135-137.

10. Butler T, Fraser GE, Beeson WL et al. (2008) Cohort profile: the Adventist Health Study-2 (AHS-2). Int J Epidemiol 37, 260-265.
11. Bes-Rastrollo M, Sabate J, Jaceldo-Siegl K et al. (2011) Validation of self-reported anthropometrics in the Adventist Health Study 2. BMC Public Health 11, 213.

12. Rizzo NS, Jaceldo-Siegl K, Sabate J et al. (2013) Nutrient profiles of vegetarian and nonvegetarian dietary patterns. J Acad Nutr Diet 113, 1610-1619.

13. Knutsen SF, Fraser GE, Linsted KD et al. (2001) Comparing biological measurements of vitamin $\mathrm{C}$, folate, $\alpha$-tocopherol and carotene with 24-hour dietary recall information in nonhispanic blacks and whites. Ann Epidemiol 11, 406-416.

14. Jaceldo-Siegl K, Knutsen SF, Sabate J et al. (2010) Validation of nutrient intake using an FFQ and repeated $24 \mathrm{~h}$ recalls in black and white subjects of the Adventist Health Study-2 (AHS-2). Public Health Nutr 13, 812-819.

15. Hollowell JG, Staehling NW, Flanders WD et al. (2002) Serum TSH, T(4), and thyroid antibodies in the United States population (1988 to 1994): National Health and Nutrition Examination Survey (NHANES III). J Clin Endocrinol Metab 87, 489-499.

16. Bahn Chair RS, Burch HB, Cooper DS et al. (2011) Hyperthyroidism and other causes of thyrotoxicosis: management guidelines of the American Thyroid Association and American Association of Clinical Endocrinologists. Thyroid 21, 593-646.

17. Procaccini C, Carbone F, Galgani M et al. (2011) Obesity and susceptibility to autoimmune diseases. Expert Rev Clin Immunol 7, 287-294.

18. Tonstad S, Butler T, Yan R et al. (2009) Type of vegetarian diet, body weight and prevalence of type 2 diabetes. Diabetes Care 32, 791-796.

19. Ardestani A \& Yazdanparast R (2007) Flavonoids as potential therapeutic agents for type 1 diabetes. Med Hypotheses 69, 955.

20. Duntas LH (2011) Environmental factors and thyroid autoimmunity. Ann Endocrinol 72, 108-113.

21. Kverka M \& Tlaskalova-Hogenova H (2013) Two faces of microbiota in inflammatory and autoimmune diseases: triggers and drugs. APMIS 121, 403-421.

22. Choi WJ \& Kim J (2014) Dietary factors and the risk of thyroid cancer: a review. Clin Nutr Res 3, 75-88.

23. Brix TH, Kyvik KO \& Hegedus L (2001) Validity of selfreported hyperthyroidism and hypothyroidism: comparison of self-reported questionnaire data with medical record review. Thyroid 11, 769-773. 